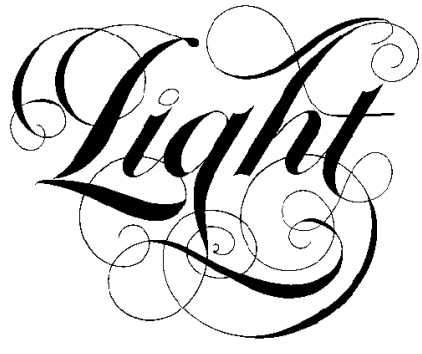

\title{
Acousto-optic superlattice modulator using a fiber Bragg grating
}

\author{
W.F.Liu, *P.St.J.Russell and L.Dong
}

*Optoelectronics Group, School of Physics, University of Bath, Bath BA2 7AY, UK

\begin{abstract}
A modulator is reported in which an extensional acoustic wave is launched along a fiber Bragg grating. The acousto-optic superlattice effect causes an enhancement in reflectivity within a narrow spectral region on both sides of the Bragg wavelength. For a fixed acoustic propagation direction, the Doppler shift can be either positive or negative, depending on whether the wavelength of the incident light lies above or below the Bragg condition. The device can function as a Bragg cell and a tunable filter.
\end{abstract}

All-fiber acousto-optic devices have potential uses as frequency shifters, multiplexers, modulators, and tunable filters. The ease with which these devices can be spliced into systems, and the consequent low insertion loss, make them an attractive alternative to pigtailed bulk Bragg cells. Previous designs of intermodal coupler include dual-mode fibers supporting an acoustic flexural wave [1,2], coupling between the polarization normal modes of a high-birefringence fiber by means of a torsional acoustic wave [3], and a high-performance device based on a four-port fused-taper null coupler [4]. All these devices share the requirement that the acoustic wavelength must match the intermodal beat length, $\mathrm{L}_{\mathrm{B}}=2 \pi / \Delta \beta$, where $\Delta \beta=\left|\beta_{2}-\beta_{1}\right|$ and $\beta_{1}$ and $\beta_{2}$ are the propagation constants of the modes.

In this Letter we describe a modulator (briefly reported for the first time in Ref. 5) in which a fiber Bragg grating is excited by an axially propagating extensional acoustic wave. The underlying principle is acousto-optic superlattice modulation (AOSLM), first proposed in 1986.[6,7] In AOSLM the counterpropagating optical modes (the Bloch waves [8]) of the fine-pitch Bragg grating are coupled by a course-pitch acoustic wave, the superposition of the two forming a superlattice. Coupling is maximum when the inter-Bloch-wave beat period matches the acoustic wavelength.

The forward-travelling (group velocity in the $+z$ direction) Bloch wave in a fiber Bragg grating can be closely approximated as a constant superposition of two coupled counterpropagating guided modes with wave vectors $\mathrm{k}$ in the form [8]

$$
k=\left\{ \pm K+\vartheta\left[1-(2 \kappa / \vartheta)^{2}\right]^{1 / 2}\right\} / 2
$$

where $\mathrm{K}=2 \pi / \Lambda$ is the Bragg grating vector and $\Lambda$ is its physical pitch. Coupling constant $\kappa$ and dephasing parameter $\vartheta$ are defined as $\kappa=M k_{d} / 4$ and $\vartheta=2 k_{o}-K$, where $k_{o}=\omega n_{o} / c$ is the average wave vector in the Bragg grating, $\mathrm{n}_{\mathrm{O}}$ is the modal phase index, and $\mathrm{M}$ is the modulation depth of the dielectric constant, i.e., $\epsilon_{\mathrm{r}}=\mathrm{n}_{\mathrm{O}}{ }^{2}(1+\mathrm{M} \cos \mathrm{Kz})$. Reversing the sign of $\vartheta$ in Eq. (1) yields the wave vectors of the complementary backward-travelling Bloch wave. The presence of two wave vectors in each Bloch wave, combined with their strong dispersion with frequency (forming a stop band in the range $-2 \kappa<\vartheta<2 \kappa)$, yields three different acousto-optic resonance conditions as opposed to one in normal fiber. Two of these conditions are at approximately the usual Brillouin frequencies $(\sim 10 \mathrm{GHz}$ at $1530 \mathrm{~nm})$. The third, which concerns us in this Letter, is indicated on the $\omega-\mathrm{k}$ diagram in Fig. 1. This condition occurs between the two forward (and the two backward) 
wave vectors of the two

Bloch waves and has an associated frequency shift that can lie between 0 and several hundred megahertz (for clarity, acoustic frequency shift $\omega_{\mathrm{s}}$ is exaggerated in Fig. 1). Note that in one-dimensional photon-photon scattering the two-vector $(\omega, \mathrm{k})$ must be conserved, i.e., $\left(\omega_{\text {out }}\right.$, $\left.\mathrm{k}_{\text {out }}\right)-\left(\omega_{\mathrm{in}}, \mathrm{k}_{\mathrm{in}}\right)=\left(\omega_{\mathrm{s}}, \mathrm{k}_{\mathrm{s}}\right) \sim$ where the frequency components must all be positive (acoustic parameters have the subscript ${ }_{\mathrm{s}}$; we ensure this by choosing the input and output two-vectors so as to make $\omega_{\text {out }}-\omega_{\text {in }}=\omega_{\mathrm{s}}>0$. The group velocity of each Bloch mode is given by $\omega / \mathrm{k}= \pm\left(\mathrm{c} / \mathrm{n}_{\mathrm{o}}\right)$ $\left[1-(2 \kappa / \mho)^{2}\right]^{1 / 2}$ which tends to zero at each stop-band edge and changes sign on opposite sides of the lines $\mathrm{k}= \pm \mathrm{K} / 2$. An intriguing feature of superlattice coupling is that the direction of the Doppler shift can be reversed. On the upper stopband branch, an acoustic wave travelling into the incident Bloch mode will produce a frequency-upshifted reflected Bloch mode. On the lower stop-band branch, however, the reflected mode will be anomalously frequency downshifted. As we show below, these predictions are confirmed by experiment.

An acoustic wave of average power $\mathrm{P}_{\mathrm{s}}$ will produce a peak strain of $\mathrm{s}_{\mathrm{O}}=$ $\left[2 \mathrm{P}_{\mathrm{s}} /\left(\mathrm{EA}_{\mathrm{gs}}\right)\right]^{1 / 2} \sim$ where $\mathrm{E}$ is Young's modulus, $\mathrm{A}$ is the fiber area, and $\mathrm{gs}$ is the acoustic group velocity. This strain field will sinusoidally modulate both the average index and the pitch of the grating. Close to the stop-band edges, where the group velocity is very small, both of these effects are significant. Further from the stop-band edges, however, pitch modulation dominates. Since the acoustic wave travels some $10^{5} \mathrm{x}$ more slowly than the light, we ignore its temporal dependence (while remembering the Doppler shift). For a strain field in the form $\mathrm{s}(\mathrm{z})=\mathrm{s}_{\mathrm{O}} \cos \left(\mathrm{k}_{\mathrm{s}} \mathrm{z}\right) \sim$ the resulting relative dielectric constant $\epsilon(\mathrm{z})$ is given by (2)

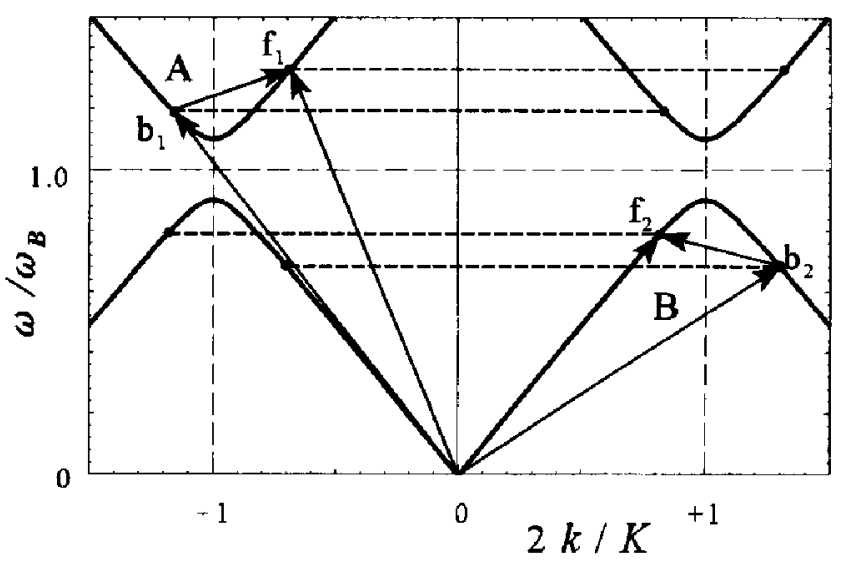

Fig. 1. Frequency wave-vector diagram for a Bragg grating. The group velocity is proportional to the slope of the curves. In case $A$, a forward-travelling acoustic wave $\left[\left(\omega_{s}, k_{s}\right)\right.$ vector shown by the arrow from $b_{1}$ to $f_{1}$ ] couples a forward-travelling Bloch wave $\left(f_{1}\right)$ into a downshifted backward one $\left(b_{1}\right)$. In case $B$, forward Bloch wave $f_{2}$ is coupled into anomalously downshifted backward Bloch wave $b_{2}$ by backward-travelling acoustic wave. The thick horizontal dashed lines join the $(\omega, k)$ points of the forward and backward fiber modes of each Bloch wave.

$$
\begin{aligned}
& \frac{\epsilon(z)-n_{o}^{2}}{M n_{o}^{2}}=\cos \left[K z+a \sin \left(k_{s} z\right)\right] \\
& =J_{o}(a) \cos K z+\sum_{n=1}^{x} J_{n}(a)\left[\cos \left(K z+n k_{s} z\right)\right. \\
& \left.\quad+(-1)^{n} \cos \left(K z-n k_{s} z\right)\right],
\end{aligned}
$$




$$
\frac{c \Delta}{2} \approx \Delta=\frac{c}{2 n_{o}}\left[\left(\frac{f_{s}}{s}\right)^{2}+\left(\frac{\kappa}{\pi}\right)^{2}\right]^{1 / 2} \approx \frac{c f_{s}}{2 n_{o} s},
$$

here $\mathrm{a}=\mathrm{Ks}_{\mathrm{O}} / \mathrm{ks}$. It is clear that a sequence of ghosts of the original fiber grating forms at spatial frequencies given by successive spatial sidebands of $\mathrm{K}$. The amplitudes of these sidebands are given, for small argument $|\mathrm{a}|<<1$, by $J_{n}(a) \approx a^{n} /\left(2^{n} n !\right)$. The AOSLM resonance occurs when the acoustic wave vector matches the wave-vector difference between two Bloch waves. This leads to the condition

where $f_{s}$ and ${ }_{s}$ are the acoustic frequency and the phase velocity and $\Delta$ and $\Delta$ are the optical frequency and wavelength shifts from the Bragg condition of the fiber grating. The approximate expression on the right-hand side of Eq. (3) is valid when the AOSLM condition occurs far from the Bragg condition. For bulk silica sis $5760 \mathrm{~m} / \mathrm{s}$, and given our experimental value of $\kappa=$ $1.3 / \mathrm{mm}$, Eq. (3) shows that AOSLM resonance will occur, e.g., at NM from the Bragg wavelength if the acoustic frequency is $15 \mathrm{MHz}(\mathrm{NB}$ :

falls when the acoustic wavelength is comparable with the fiber radius [9]). It can be shown that the coupling constant between the counterpropagating Bloch waves at the first sideband in Eq. (2) is given approximately by $\kappa_{1}=$ $\kappa J_{1}\left(\mathrm{Ks}_{0} / \mathrm{k}_{\mathrm{s}}\right) \approx \kappa \mathrm{Ks}_{0} /\left(2 \mathrm{k}_{\mathrm{s}}\right)$. Figure 2 shows the experimental setup. The fiber grating was written with a phase mask in a boron-codoped germanosilicate fiber (N.A., 0.115; cutoff, $1300 \mathrm{~nm})$. The UV source was an ArF excimer laser at $193 \mathrm{~nm}$. The grating was $3 \mathrm{~mm}$ long, with a

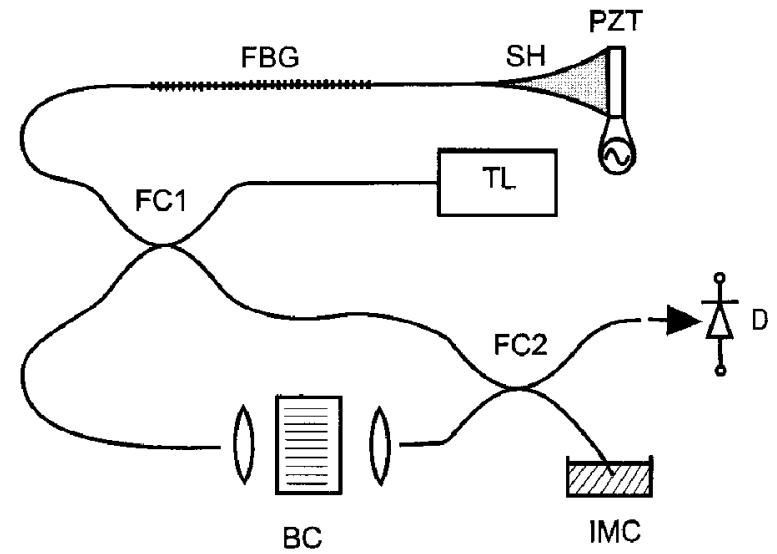

Fig. 2. Experimental setup for monitoring the response of AOSLM. Light from a tunable single-frequency diode laser (TL) is divided at a fused taper coupler (FC1), one half going to the fiber Bragg grating (FBG) and the other to a bulk Bragg cell (BC). The light reflected from the AOSLM is combined with the frequency-shifted light from the Bragg cell at a second coupler (FC2), and the mixed signal is detected at a square-law detector (D); the redundant light in the second arm of FC2 is eliminated in an index-matching cell (IMC). A piezoelectric transducer (PZT) and a fused-silica horn (SH) are used to excite the AOSLM.

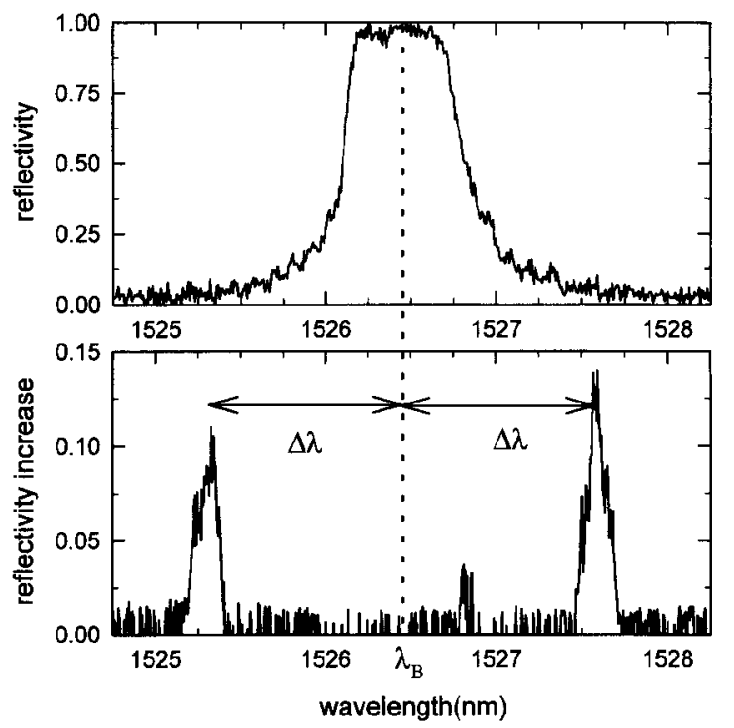

Fig. 3. Reflectivity spectrum of the undistributed Bragg grating (top) and the spectrum of the enhancement in reflectivity due to the acoustic wave (bottom). 
bandwidth of $0.7 \mathrm{~nm}$, a Bragg wavelength of $1526.5 \mathrm{~nm}$, and a coupling constant $\kappa=1.3 / \mathrm{mm}$. A piezoelectric transducer, bonded to a silica horn and driven by a rf signal generator, was used as the source of acoustic waves. The diameter of the silica horn was tapered from $3 \mathrm{~mm}$ to $125 \mu \mathrm{m}$ over a distance of $\sim 7 \mathrm{~cm}$. One end of the fiber grating was spliced directly to the horn. To test whether the light reflected from the grating is upshifted or downshifted, the light was mixed at a square-law detector with light frequency shifted in a conventional Bragg cell. We found that the interface loss between the transducer and the silica horn is a very important factor in obtaining efficient coupling of the longitudinal acoustic wave to the fiber grating. The light was detected at one of the output ports of $3 \mathrm{~dB}$ coupler FC2, with the remaining port being immersed in index-matching oil.

The reflectivity spectrum of the undisturbed Bragg grating and the spectrum of the enhancement in reflectivity due to the acoustic wave are plotted in Fig. 3. AOSLM is observed on both sides of the stop band. The acoustic frequency was $8.02 \mathrm{MHz}$, and the electrical drive power to the transducer was $350 \mathrm{~mW}$. The wavelengths of AOSLM reflection were at $1525.3 \mathrm{~nm}$ on the short-wavelength side and $1527.6 \mathrm{~nm}$ on the long-wavelength side. The magnitude of the wavelength shift $\Delta$ from Bragg wavelength is in each case approximately $1.15 \mathrm{~nm}$, and the bandwidth of the AOSLM effect is $\sim 0.2 \mathrm{~nm}$. In a series of measurements of wavelength shift $\Delta$ as a function of acoustic frequency (4 to $15.2 \mathrm{MHz}$ in steps of $1 \mathrm{MHz}$ ) the agreement between theory [Eq. (3)] and experiment was better than a few percent. The results showed that AOSLM could be used to form a tunable filter with a tuning rate of $0.15 \mathrm{~nm} / \mathrm{MHz}$.

The results of the heterodyne experiment are shown in Fig. 4. A tunable single-frequency laser was used, and the acoustic drive frequency was $8 \mathrm{MHz}$. On the short-wavelength side of the Bragg condition the reflected light was frequency upshifted (conventional Doppler effect), whereas on the long-wavelength side it was downshifted (anomalous Doppler shift) as predicted by theory.

Since the spatial sidebands produced by the acoustic wave [Eq. (2)] can be regarded as weak ghosts of the strong permanent Bragg grating, the FWHM bandwidth of the AOSLM effect is expected to be that of a weak Bragg grating of the same length, namely, $\Delta{ }_{\text {opt }}$ $=1.39^{2} /\left(\pi \operatorname{Ln}_{\mathrm{o}}\right)$ where $\mathrm{L}$ is the fiber length and the numerical factor relates to $\operatorname{sinc}^{2}(1.39)=1 / 2$. For $L=3 \mathrm{~mm}$ at $=$ $1530 \mathrm{~nm}$ this equation predicts $\Delta{ }_{\text {opt }}=$ $0.23 \mathrm{~nm}$, which is in reasonable agreement with the experimental results.

For small acoustic powers, it can be shown that the efficiency of conversion can be approximated by $\approx\left(\kappa_{1} \mathrm{~L}\right)^{2}=$ $\left[\kappa \mathrm{LJ}_{1}\left(\mathrm{Ks}_{\mathrm{o}} / \mathrm{k}_{\mathrm{s}}\right)\right]^{2}$. For the parameters in

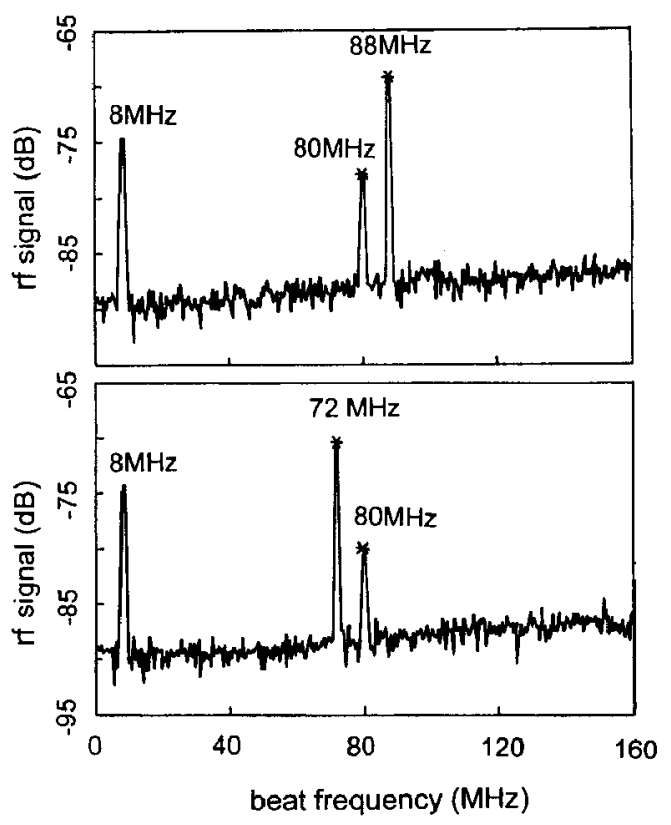

Fig. 4. RF spectra at the detector for sidelobes at $>_{B}$ (top) and $<_{B}$ (bottom). The peak at $8 \mathrm{MHz}$ is caused by beating between the unshifted and the shifted Bragg grating reflections. The presence of a band on the low-frequency side of the $80 \mathrm{MHz}$ reference signal indicates that the AOSLM signal is frequency upshifted. 
our experiment $\sim 15 \%$, and using the relationship given in the paragraph above Eq. (2) to relate acoustic power to $\mathrm{s}_{\mathrm{O}}$, it can be shown that of the $350 \mathrm{~mW}$ electrical drive power, only $57 \mathrm{~mW}$ is actually reaching the Bragg grating as acoustic power. Since the efficiency scales linearly with the power and as the square of length, there is clearly scope for improving the AOSLM efficiency by better acoustic transducer design, increasing the grating length, or using thinner fibers. Some advantages would result if the core was offset from the centre of the fiber and flexural waves were used.

Substantial improvements are expected with better acoustic transducer design, a longer fiber grating, and thinner fiber.

\section{References}

1. $\quad$ B.Y.Kim, J.N.Blake, H.E.Engan and H.J.Shaw, Opt. Lett. 11, 389 (1986).

2. J.N.Blake, B.Y.Kim, H.E.Engan and H.J.Shaw, Opt. Lett. 12, 281 (1987).

3. M.Berwick, C.N.Pannell, P.St.J.Russell and D.A.Jackson, Electron. Lett. 27, 713 (1991).

4. T.A.Birks, S.G.Farwell, P.St.J.Russell and C.N.Pannell, Opt. Lett. 19, 1964 (1994); erratum, Opt. Lett. 21, 231 (1996).

5. W.F.Liu, P.St.J.Russell, D.O.Culverhouse and L.Reekie, in Conference on Lasers and Electro-Optics, Vol. 9 of 1996 OSA Technical Digest Series (Optical Society of America, Washington, D.C., 1996), pp. 243-244.

6. P.St.J.Russell, Phys. Rev. Lett. 56, 596 (1986).

7. P.St.J.Russell, J. Appl. Phys. 59, 3344 (1986).

8. $\quad$ P.St.J.Russell, J. Mod. Opt. 38, 1599 (1991).

9. H.Kolsky, Stress Waves in Solids (Oxford U. Press, London, 1953), Chap III. 